\title{
107. A New Method for Downward Continuation of Two-Dimensional Gravity Distribution
}

\author{
By Hiroo KanamoRI \\ Geophysical Institute, University of Tokyo \\ (Comm. by Chuji Tsubor, M.J.A., Sept. 12, 1963)
}

The merit of the $\sin x / x$ method which was introduced by. Y. Tomoda and $\mathrm{K}$. $\mathrm{Aki}^{13}$ for downward continuation of one-dimensional gravity distribution lies in its simplicity of application as well as in its ciearness of physical meaning, especially from the standpoint of the theory of spectrum.

In this $\sin x / x$ method, gravity anomalies which are considered to be one-dimensional in horizontal distribution are represented by anomaly values at equidistant grid points. The anomaly value at any $x$ between the grid points is considered to be given by a function

$$
\sum_{i=-\infty}^{\infty} \Delta g_{i} \frac{\sin \left(x-\xi_{i}\right)}{\left(x-\xi_{i}\right)}
$$

where $x-\xi_{i}$ is the distance between the point $x$ and the $i$-th grid point at which the anomaly is $\Delta g_{i} . \quad x$ and $\xi_{i}$ are both to be given in the circular measure, $\pi$ corresponding to the grid point spacing.

From the spectral point of view, this amounts to mean that the original continuous gravity data are put into a special filter and the output from it is used as materials for interpretation. Since

$$
\frac{\sin x}{x}=\int_{0}^{1} \cos m x d m,
$$

the operation spectrum of the filter is a square in form (Fig. 1), component waves in the input which are higher than $m=1$ in frequency (wave length shorter than $2 \pi$ ) being completely cut off by it.

$\sin x / x$ is the result of filteration of such a gravity anomaly distribution that is 1 at $x=0$ and 0 at all other grid points. The mass distribution $M(x)$ at a depth $d$ that will produce this anomaly distribution is given by

$$
\begin{aligned}
M(x) & =\frac{1}{2 \pi k^{2}} \int_{0}^{1} \cos m x \cdot \exp (m d) d m \\
& =\frac{1}{2 \pi k^{2}} \frac{1}{x^{2}+d^{2}}[x \sin x \cdot \exp d+d \cos x \cdot \exp d-d] \equiv \frac{1}{2 \pi k^{2}} \Phi(x) .(1)
\end{aligned}
$$

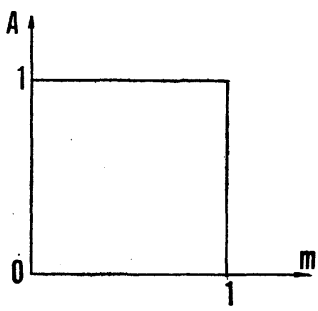

At $x=p \pi(p$; integer $), \Phi(x)$ becomes 


$$
\Phi(p \pi)=\frac{d}{p^{2} \pi^{2}+d^{2}}( \pm \exp d-1) \quad \begin{aligned}
& +: p \text { even } \\
& -: p \text { odd }
\end{aligned}
$$

$\Phi$ is easy to evaluate numerically. Taking anomaly values at other grid points into account,

$$
\frac{1}{2 \pi k^{2}} \sum_{p=-\infty}^{+\infty} \Delta g_{i+p} \Phi(p \pi)
$$

will at once give the mass right below the $i$-th grid point.

This method has been very useful for interpreting gravity anomaly distribution in several regions which may well be regarded as onedimensional. C. Tsuboi ${ }^{2}$ extended this method for upward continuation of gravity distribution also. The success depends very much on the mathematical simple form of $\Phi$. To calculate $\Phi$ for a particular value of $d$ which is appropriate to any actual problem is simply a desk calculator work.

It is highly desirable to extend this method so as to be used for studying gravity anomalies which are two-dimensional in distribution. For that purpose, numerical evaluation of the integral

$$
\int_{0}^{1} \int_{0}^{1} \cos m x \cdot \cos n y \cdot \exp \left(\sqrt{m^{2}+n^{2}} \cdot d\right) d m d n
$$

is required corresponding to the integral (1) in one-dimensional case. Unfortunately no closed form expression of this integral (2) is available, so that each time when $d$ is given in actual problem, numerical evaluation of this integral for that $d$ should be carried out by means of electronic or other high speed computers unless comprehensive numerical tables of this integral for a variety of $d$ are available. Although Tsuboi, Oldham and Waithman ${ }^{3)}$ and Shimazu ${ }^{4)}$ published the values of the integral for $d=\pi / 2$ and $d=\pi$, these are too short to be of wide use.

The filter to be used in this case is a cube in form (Fig. 2), corresponding to a square filter which is used in one-dimensional case. There is no compelling reason, however, why this cubic filter should be the only one to be used. It is proposed here to deform

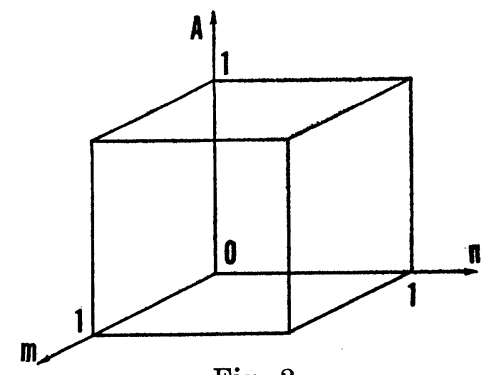

Fig. 2 the operation spectrum of the filter slightly by a factor $A(m, n)$ so that the integral

$$
\Phi=\int_{0}^{1} \int_{0}^{1} A(m, n) \cdot \cos m x \cdot \cos n y \cdot \exp \left(\sqrt{m^{2}+n^{2}} \cdot d\right) d m d n
$$

can be expressed in a closed form. $A(m, n)$ may be such that the amplitude reduction will be more or less larger for higher component waves and for larger $d$ than for lower component waves and for 
smaller $d$, though this will mean a slight loss of informations provided by such reduced components. To deform the spectrum of the filter slightly means to use an interpolation formula which differs slightly from the $\sin x / x \cdot \sin y / y$ formula. There can be several such deformations but the one proposed here is as follows:

$$
\begin{aligned}
A(m, n) & =\exp \left(n-\sqrt{m^{2}+n^{2}}\right) d, & & m \leqq n \leqq 1 \\
& =\exp \left(m-\sqrt{m^{2}+n^{2}}\right) d, & & n \leqq m \leqq 1 \\
& =0 & & \text { otherwise. }
\end{aligned}
$$

Since on $m$ and $n$ axis for which $n=0$ and $m=0$ respectively, the new modified filter has exactly the same spectrum as the unmodified one. At $m=1, n=1$

$$
A(1,1)=\exp (1-\sqrt{2}) d=\exp (-0.414 d)
$$

In practical problems, $d$ seldom exceeds $\pi / 2$ in which case

$$
A(1,1)=0.522 \text {. }
$$

Component waves in the input for which $\sqrt{m^{2}+n^{2}}$ is large are somewhat more strongly reduced in amplitude by this new modified filter than by the previous cubic filter.

The problem is now to evaluate the integral

$$
\Phi=\int_{0}^{1} \int_{0}^{1} A(m, n) \cdot \cos m x \cdot \cos n y \cdot \exp \left(\sqrt{m^{2}+n^{2}} \cdot d\right) d m d n .
$$

Remembering the definition of $A(m, n)$ as given by (3), $\Phi$ can be integrated into the following closed form:

$$
\begin{aligned}
\Phi= & \frac{1}{\left\{d^{2}+(x+y)^{2}\right\}\left\{d^{2}+(x-y)^{2}\right\}}\left[d\left(d^{2}-x^{2}+y^{2}\right) \cos y \frac{\sin x}{x} \cdot e^{d}\right. \\
& +d\left(d^{2}+x^{2}-y^{2}\right) \cos x \frac{\sin y}{y} e^{d}-2 d^{2} \cos x \cos y e^{d} \\
& \left.+\left\{d^{2}\left(x^{2}+y^{2}\right)+\left(x^{2}-y^{2}\right)^{2}\right\} \frac{\sin x}{x} \frac{\sin y}{y} e^{d}+2 d^{2}\right] .
\end{aligned}
$$

At the grid points where

$\Phi$ becomes

$$
x= \pm m \pi, y= \pm n \pi \quad(m=0,1,2, \cdots, n=0,1,2, \cdots)
$$

1) $x=0, y=0$,

$$
\Phi=\frac{2}{d^{2}}\left\{e^{d}(d-1)+1\right\}
$$

2) $x= \pm m \pi(m \neq 0), y=0$,

$$
\Phi=\frac{d}{\left(d^{2}+m^{2} \pi^{2}\right)^{2}}\left\{(-1)^{m} e^{d}\left(d^{2}+m^{2} \pi^{2}-2 d\right)+2 d\right\},
$$

3) $x=0, y= \pm n \pi(n \neq 0)$,

$$
\Phi=\frac{d}{\left(d^{2}+n^{2} \pi^{2}\right)^{2}}\left\{(-1)^{n} e^{d}\left(d^{2}+n^{2} \pi^{2}-2 d\right)+2 d\right\},
$$

4) $x= \pm m \pi(m \neq 0), y=n \pm \pi(n \neq 0)$,

$$
\Phi=\frac{2 d^{2}}{\left\{d^{2}+(m+n)^{2} \pi^{2}\right\}\left\{d^{2}+(m-n)^{2} \pi^{2}\right\}}\left\{1-(-1)^{m+n} e^{d}\right\}
$$




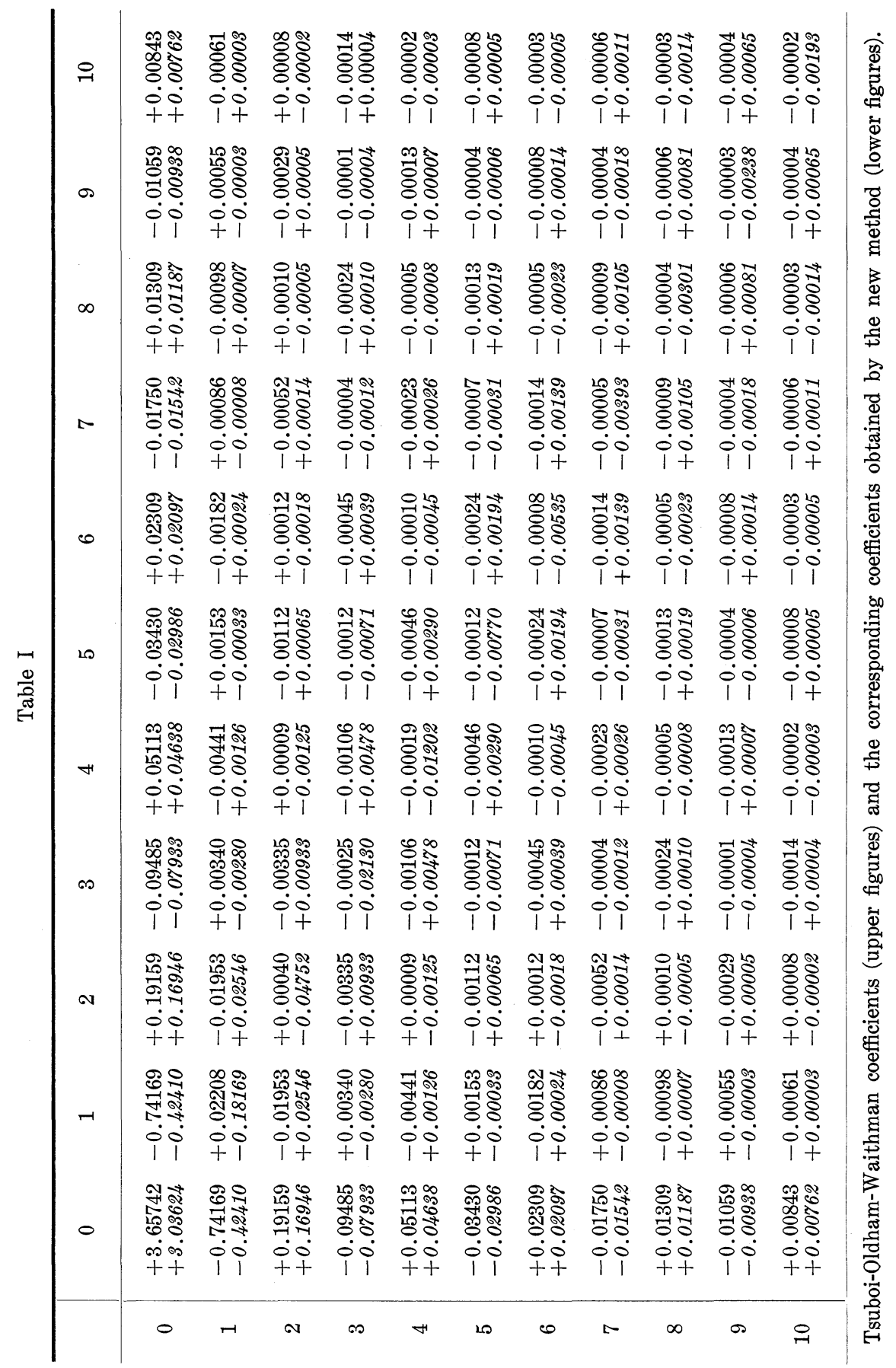


Using this $\Phi$, the mass at a depth $d$ below the point $x=i \pi, y=j \pi$ which will produce a given gravity anomaly distribution $\Delta g(i \pi, j \pi)$ is given by

$$
M(i \pi, j \pi)=\frac{1}{2 \pi k^{2}} \sum_{m=-\infty}^{+\infty} \sum_{n=-\infty}^{+\infty} \Phi(m, n) \cdot \Delta g\{(i+m) \pi,(j+n) \pi\} .
$$

Once $d$ is fixed appropriately for each actual problem, nothing more remains to be done than arithmetical computations of $\Phi$ values and summations of $\Phi \cdot \Delta g$ by means of a desk calculator. This applies for any value of $d$ and represents a great advantage over the use of the unmodified cubic filter for which no closed form expression of $\Phi$ is available so that each time when a particular value of $d$ is given, electronic computer is required for obtaining $\Phi$ value for that depth.

$\Phi$ values for $d=\pi / 2$ as calculated by the present method and those previously calculated by Tsuboi, Oldham and Waithman ${ }^{3)}$ by means of electronic computer using the unmodified filter are compared in Table I.

It is evident that this new method presented here works particularly well if 1 ) component waves for which $\sqrt{m^{2}+n^{2}}$ is around 1 are small in amplitude in the original gravity anomaly distribution, 2) the value of $d$ is small as compared with the grid spacing, and 3 ) the original gravity is nearly one dimensional in distribution. Even when these conditions are not strictly satisfied, the new method can still effectively be used with an understanding that higher component waves in the original gravity anomaly distribution are more or less strongly reduced.

Examples of application of the present method and comparisons of the results obtained by the old and new method will be given in another article.

The author gratefully acknowledges Emeritus professor Chuji Tsuboi who kindly directed the author's attention to the present problem.

\section{References}

1) Y. Tomoda and K. Aki: Proc. Japan Acad., 31, 443-448 (1955).

2) C. Tsuboi: Reports of the Institute of Geodesy, Photogrammetry and Cartography, Ohio State Univ., 3, 1-18 (1959).

3) C. Tsuboi, C. H. G. Oldham and V. B. Waithman: J. Phys. Earth, 6, 7-13 (1958).

4) Y. Shimazu: Pub. Dominion Obs., 26, 323-354 (1962). 